\title{
Activated Natural Killer Cells in Combination with Anti-GD2 Antibody Dinutuximab Improve Survival of Mice after Surgical Resection of Primary Neuroblastoma
}

\author{
Wesley E. Barry ${ }^{1,2}$, Jeremy R. Jackson ${ }^{2}$, Grace E. Asuelime ${ }^{2}$, Hong-Wei Wu ${ }^{3}$, Jianping \\ Sun $^{3}$, Zesheng Wan ${ }^{3}$, Jemily Malvar ${ }^{3}$, Michael A. Sheard ${ }^{3}$, Larry Wang ${ }^{4}$, Robert C. Seeger ${ }^{3}$, \\ and Eugene S. Kim ${ }^{1,2}$ \\ ${ }^{1}$ Department of Surgery, Keck School of Medicine, University of Southern California, Los Angeles, \\ CA \\ ${ }^{2}$ Division of Pediatric Surgery, Children's Hospital Los Angeles, Los Angeles, CA \\ ${ }^{3}$ Division of Hematology, Oncology, and Blood and Marrow Transplantation, Children's Hospital \\ Los Angeles, Keck School of Medicine, University of Southern California \\ ${ }^{4}$ Department of Pathology and Laboratory Medicine, Children's Hospital Los Angeles, Keck \\ School of Medicine, University of Southern California
}

\begin{abstract}
Purpose: Immunotherapy of neuroblastoma that remains after myeloablative chemotherapy with anti-GD2 antibody dinutuximab has increased the two-year event-free and overall survival of highrisk neuroblastoma patients; however, $40 \%$ of patients develop recurrent disease during or after this treatment. To determine the potential of such antibody-based immunotherapy earlier in treatment, a mouse model was developed in which surgical resection of the primary tumor was followed by therapy of residual disease with dinutuximab combined with ex vivo-activated human natural killer (aNK) cells.
\end{abstract}

Experimental Design: The effect of combining dinutuximab with human aNK cells was determined in vitro with cellular cytotoxicity and Matrigel invasion assays. The in vivo efficacy of dinutuximab and aNK cells against neuroblastoma was assessed following resection of primary tumors formed by two cell lines or a patient derived xenograft (PDX) in immune deficient NODscid gamma mice.

Results: In vitro, the combination of aNK cells and dinutuximab caused cytotoxicity and decreased invasiveness of three human neuroblastoma cell lines. Treatment of mice with dinutuximab combined with aNK cells after surgical resection of primary intrarenal tumors formed by two cell lines or a PDX decreased tumor cells in liver and bone marrow as evaluated by histopathology and bioluminescence imaging. Survival of mice after resection of these tumors was

Correspondence: Eugene S. Kim, MD; Children's Hospital Los Angeles; 4650 Sunset Blvd, MS 100, Los Angeles, CA 90027, Tel: (323) 361-8332; eugeneskim@ chla.usc.edu.

Conflict of Interest: The authors declare no potential conflicts of interest. 
most significantly increased by treatment with dinutuximab combined with aNK cells compared to that of untreated mice.

Conclusions: The combination of dinutuximab and adoptively transferred human aNK cells following surgical resection of primary neuroblastomas significantly improves survival of immune deficient mice.

\section{INTRODUCTION}

Neuroblastoma (NB) is a malignancy of neuroectodermal origin and is the most common extracranial solid tumor of childhood (1-4). Despite improvements in multimodal therapy, the 5-year event-free survival (EFS) for patients with high-risk disease remains approximately $45 \%(2,3,5-8)$. Nearly $80 \%$ of patients respond to induction therapy, which includes chemotherapy and surgical resection of the primary tumor (8). However, this initial therapy requires improvement as only $22 \%$ and $56 \%$ of patients achieve complete and partial clinical responses (8). Immunotherapy with anti-disialoganglioside (GD2) monoclonal antibody (dinutuximab) could improve the efficacy of induction therapy since its use after myeloablative therapy supported by autologous hematopoietic stem cell transplantation has increased the 2-year EFS and overall survival (9). We have previously shown that cryopreserved, ex vivo propagated and activated natural killer (aNK) cells maintain their ability to induce antibody-dependent cellular cytotoxicity (ADCC) when combined with dinutuximab and improve survival of immune deficient mice with disseminated NB (10).

The impact of immunotherapy with dinutuximab combined with adoptively transferred aNK cells on NB cells remaining after surgical resection of the primary tumor has yet to be examined. We developed a model in immune deficient NOD-scid gamma (NSG) mice that simulates a clinical scenario in which immunotherapy is given following surgical resection. The primary tumor is established by injecting human NB cells into the kidney of NSG mice, and it is resected after growing for seven days. Although resection is grossly complete, untreated mice have tumor cells that are detectible by bioluminescence imaging at the primary site one day after resection and in liver and bone marrow by imaging and histopathology within three to four weeks (11). We show that dinutuximab combined with adoptively transferred aNK cells following surgical resection decreases NB growth in liver and bone marrow and increases survival of mice.

\section{MATERIALS AND METHODS}

\section{NB cell lines and patient-derived xenograft}

CHLA-136, CHLA-255, and SH-SY5Y human NB cell lines as well as NB patient-derived xenograft (PDX) COG-N-415x cells were derived from patients with progressive disease (12-17). CHLA-136 and CHLA-255 cells were obtained from the Children's Oncology Group (COG) Cell Culture and Xenograft Repository (www.COGcell.org). SH-SY5Y cells were obtained from American Type Culture Collection (ATCC). CHLA-136 cells have a high level of GD2 expression (Supplementary Fig. S1) and have genomic amplification of MYCN(14). CHLA-255 cells have a high level of GD2 expression and express c-MYC protein, thereby representing high-risk, undifferentiated/poorly differentiated NB lacking 
MYCN proto-oncogene amplification (18-20). Notably, patients expressing MYCN or cMYC protein detected by immunofluorescence have similar and significantly low survival (20). SH-SY5Y cells have a medium level of GD2 expression and are $M Y C N$-non-amplified (21). COG-N-415× PDX cells have a medium to high level of GD2 expression and have amplification of $M Y C N$ and mutation of $A L K$ (F1174L) (provided by Dr. C. Patrick Reynolds, www.COGcell.org). These three cell lines and PDX represent the heterogeneity of high-risk human NBs. The firefly luciferase (Fluc) gene was transduced into SH-SY5Y (SHSY5Y-Fluc), CHLA-136 (CHLA-136-Fluc) cells and CHLA-255 (CHLA-255-Fluc) cells using a lentivirus vector, as previously described $(10,17)$.

\section{aNK cells, reagents, and cell culture}

aNK cells from healthy human donors were propagated and activated ex vivo by incubating peripheral blood mononuclear cells (PBMC) in RPMI1640 and 10\% heat-inactivated fetal bovine serum (FBS; Omega Scientific, Tarzana, CA; Catalog no. FB-02) containing human IL-2 (10 ng/ml, 100 U/mL; PeproTech, Rock Hill, NJ; Catalog no. 200-02) and irradiated (100 Gy) K562-mbIL21 feeder cells genetically engineered to express immunostimulatory molecules including CD137 ligand and membrane-bound IL-21, as previously described (10, $17,22)$. At day 14 , aNK cells were cryopreserved in aliquots. Upon thawing, aNK cells were either allowed to recover for in vitro assays by culturing in RPMI-1640 and 10\% FBS with IL-2 for two days or were thawed and immediately injected intravenously into mice.

The human NB cell line SH-SY5Y-Fluc was maintained in RPMI-1640 (Corning, Manassas, VA; Catalog no. 10-040-CV). Human NB cell lines CHLA-136-Fluc and CHLA-255-Fluc were maintained in Iscove's Modified Dulbecco's medium (IMDM) (University of Southern California Stem Cell Core, Los Angeles, CA). All media included 10\% FBS and 2 mmol/L L-glutamine (Gibco by Life Technologies, Grand Island, NY; Catalog no. 25030-081). Cell lines were maintained at $37^{\circ} \mathrm{C}$ in $5 \% \mathrm{CO}_{2}$ until $80 \%$ confluence was reached, and then they were harvested using $0.5 \%$ trypsin-EDTA (Corning, Manassas, VA; Catalog no. 25-052$\mathrm{CL})$. All cell lines were screened routinely for mycoplasma, and donor-cell identity was authenticated by short tandem repeat multiplex assay using the AmpFLSTR ${ }^{\mathrm{TM}}$ Identifiler $^{\mathrm{TM}}$ PCR Amplification Kit (Applied Biosystems, Foster City, CA; Catalog no. 4322288).

The following reagents were used: dinutuximab (United Therapeutics, Silver Spring, MD), recombinant human interleukin-2 (IL-2) (PeproTech, Rock Hill, NJ; Catalog no. 200-02), and recombinant human interleukin-15 (IL-15) (PeproTech; Catalog no. 200-15).

\section{Flow cytometry}

Surface staining for GD2 was performed on SH-SY5Y-Fluc, CHLA-136-Fluc, CHLA-255Fluc and COG-N-415× cells. Briefly, cells were washed twice in fluorescence-activated cell sorting (FACS) buffer (PBS with 5\% bovine serum albumin (Fisher Scientific SH3057402) and centrifuged for 8 minutes at $100 \times \mathrm{g}$. Fc-receptors were blocked by incubation in human Fc-blocker for 10 min at $4^{\circ} \mathrm{C}$ (Human True Stain FcX, Biolegend 422302), followed by incubation with anti-human GD2 antibody (BioLegend 357306) and isotype-matched irrelevant control (BD Pharmingen 340754) for $1 \mathrm{hr}$ in the dark at $4^{\circ} \mathrm{C}$. Cells were then washed twice in FACS buffer, and DAPI was added $(0.5 \mathrm{ng} / \mathrm{ml}$ final concentration) to each 
tube. Flow cytometry was conducted using a LSR II flow cytometer (BD Biosciences), and BD FACSDiva $^{\mathrm{TM}}$ software was used to collect and analyze data. Experiments were repeated a minimum of three times. Ratios for median fluorescence intensity (MFI) index were calculated as follows: (MFI of viable cells stained with specific antibody / MFI of viable cells stained with isotype-matched irrelevant antibody).

\section{Cytotoxicity assay}

Frozen K562.mbIL21-expanded aNK cells were cultured in 10\% FBS containing RPMI-1640 with $100 \mathrm{U} / \mathrm{ml}$ of IL-2 for $48 \mathrm{hr}$. For the cytotoxicity assay, basal media containing 2\% FBS was used. NB cell lines SH-SY5Y-Fluc, CHLA-136-Fluc and CHLA-255-Fluc were washed, resuspended in cell culture medium, and incubated with Invitrogen's calcein-AM (Thermo Fisher Scientific, Pittsburgh, PA; Catalog no. C1429) for 30 minutes at $37^{\circ} \mathrm{C}$ in $5 \% \mathrm{CO}_{2}$. The fluorescence of the calcien-AM seen by microscopy is directly proportional to the number of viable NB cells (23). NB cells were washed, and $3 \times 10^{4}$ cells were seeded per well in a 96 -well plate. After $5 \mathrm{~min}, 1.5 \times 10^{4}$ aNK cells, treated with IL-2 as described above, were added to cultures with NB cells at an effector:target (E:T) ratio of 1:2. To wells containing aNK cells, $100 \mathrm{U} / \mathrm{mL}$ of IL-2 was added. Dinutuximab was added at a concentration of $100 \mathrm{ng} / \mathrm{mL}$. The plate was incubated at $37^{\circ} \mathrm{C}$ in 5\% $\mathrm{CO}_{2}$ for six hours and survival of NB cells was assessed using a digital imaging microscopy system, (DimScan, available from BioImaging Solutions, Inc., San Diego, CA). Relative tumor cell survival was then calculated: (fluorescence of treated, Calcein-AM labeled cells/ fluorescence of untreated, calcein-AM labeled cells). aNK cells were not labeled and therefore did not contribute to the fluorescent signal. Each condition contained six replicates and each experiment was performed a minimum of two times.

\section{Matrigel invasion assay}

Frozen K562.mbIL21-expanded human aNK cells were cultured in 10\% FBS-RPMI-1640 with $100 \mathrm{U} / \mathrm{ml}$ of IL-2 for 48 hours. For this assay, NB cell lines SH-SY5Y-Fluc, CHLA-136-Fluc and CHLA-255-Fluc were pre-treated using basal media containing $1 \%$ FBS in T-25 flasks for $6 \mathrm{hrs}$ with (a) $100 \mathrm{ng} / \mathrm{ml}$ dinutuximab, (b) aNK cells plus $100 \mathrm{U} / \mathrm{mL}$ of IL-2, or (c) $100 \mathrm{ng} / \mathrm{mL}$ dinutuximab and aNK cells with $100 \mathrm{U} / \mathrm{mL}$ IL-2. Following treatment, flasks were washed twice with warm PBS (Corning, Manassas, VA; Catalog no. 21-031-CV) and allowed to recover for $16 \mathrm{hr}$ in basal media containing 1\% FBS. Biocoat 24-well Matrigel Invasion Chambers (Corning, Corning, NY; Catalog no. 354480) were allowed to equilibrate at room temperature for $10 \mathrm{~min}$, followed by re-hydration at $37^{\circ} \mathrm{C}$ for $2 \mathrm{hr}$. Viable cells were counted using trypan blue exclusion, and $1 \times 10^{5} \mathrm{NB}$ cells were seeded in the top well of the chamber and incubated for 72 hours in $5 \% \mathrm{CO} 2$ at $37^{\circ} \mathrm{C}$. Migrated cells were visualized using Fisher HealthCare ${ }^{\mathrm{TM}}$ PROTOCOL Hema $3^{\mathrm{TM}}$ Manual Staining System and Stat Pack (Thermo Fisher Scientific, Pittsburgh, PA; Catalog no. 22-123869). Images were captured using a Zeiss Axioplan microscope and the Spot Advanced software package (version 4.0.9, Spot Imaging, Inc., Sterling Heights, MI). Cells that migrated to the lower chamber were quantified using Image J software (version 1.50i, National Institutes of Health, Bethseda, MD). Independent experiments were performed a minimum of 3 times for each cell line. 


\section{Surgical resection of human neuroblastoma and treatment of NOD-scid gamma (NSG) mice}

Six to eight week old NSG mice, which lack NK, B, and T cells, were bred in-house and genotyped for colony maintenance. These mice were used for all in vivo experiments and cared for under pathogen-free conditions in accordance with the Institutional Animal Care and Use Committee of Children's Hospital Los Angeles (IACUC \#363-17). NB cell viability before injection was assessed using trypan blue staining, and cells were washed and suspended in sterile phosphate buffered saline (PBS) (Corning, Manassas, VA; Catalog no. 21-031-CV) at a concentration of $1 \times 10^{6} \mathrm{NB}$ per $0.1 \mathrm{ml} .1 \times 10^{6}$ CHLA-136-Fluc, CHLA-255-Fluc or COG-N-415 $\times$ cells, which were $>90 \%$ viable, were injected into the left kidney of NSG mice. After 7 days, complete surgical resection of the xenografted kidney was performed.

After surgery, mice were randomized into four treatment groups: (a) control, (b) dinutuximab alone, (c) aNK cells alone or (d) the combination of aNK cells and dinutuximab. All mice that received dinutuximab were administered $15 \mu \mathrm{g}\left(\sim 2.14 \mathrm{mg} / \mathrm{m}^{2}\right)$ by intravenous tail vein injection twice a week for 4 weeks for a cumulative dose of 17.1 $\mathrm{mg} / \mathrm{m}^{2}$, starting 2 days following surgical resection. This cumulative dose approximates the daily dose administered to patients $\left(17.5 \mathrm{mg} / \mathrm{m}^{2}\right.$, range $\left.10-25 \mathrm{mg} / \mathrm{m}^{2}\right)$ and is approximately one-fourth the cumulative monthly dose given to patients. Mice received aNK cells $\left(1 \times 10^{7}\right)$ by tail vein injection twice a week for 4 weeks starting 2 days following surgical resection. This dose is in the range of doses being used in ongoing clinical trials NCT03420963, NCT02763475 and NCT02573896. Mice receiving aNK cells were given IL-2 ( $2 \mu \mathrm{g} / \mathrm{mouse}$; $2 \times 10^{4} \mathrm{U} /$ mouse $)$ and IL-15 (5 $\mu \mathrm{g} /$ mouse; $1 \times 10^{5} \mathrm{U} /$ mouse $)$ via intraperitoneal injection at the same time as injection of aNK cells.

\section{Assessment of residual neuroblastoma by bioluminescence imaging and histopathology}

Bioluminescence imaging (Xenogen IVIS Lumina XR System, Caliper Life Sciences, Waltham, MA) was used to quantify the overall burden of disease weekly beginning two days after surgical resection of the left kidney, which was performed seven days after tumor cell injection. Total flux (photons/second) of the entire mouse after injection of CHLA-136Fluc or CHLA-255-Fluc cells was quantified using Living Image software (Living Image 4.3.1, Caliper Life Sciences, Waltham, MA) and compared to pre-treatment baseline. Incidence of distant residual tumors was determined by the presence of bioluminescence within the femurs or liver and confirmed by histopathology. Mice were euthanized when IACUC criteria for euthanasia were met, which included massive tumor burden from recurrent disease, poor eating, grooming, paralysis, and/or lethargy.

At the time of sacrifice, liver tissue and femurs from all mice were formalin-fixed and stored in $75 \%$ ethanol at $4{ }^{\circ} \mathrm{C}$. Specimens underwent serial dehydration in ethanol followed by immersion in xylene and paraffin embedding. All tissues were cut into $5 \mu \mathrm{m}$ sections and stained with hematoxylin and eosin (H\&E) by the Department of Pathology at Children's Hospital Los Angeles. Two sections of liver and a single longitudinal section of both femurs were evaluated from each mouse. The presence of morphologically identifiable NB cells in 
these sections was determined in a blinded manner by Dr. L. Wang, attending pathologist, who specializes in neuroblastoma.

\section{Statistical analyses}

Data for the in vitro cytotoxicity and Matrigel invasion assays were compared using an ANOVA and Tukey's HSD post-host test. The presence of NB in liver and bone marrow, assessed as described above, was analyzed using Fisher's exact test. Weekly bioluminescent flux measurements were adjusted to baseline observations and the area under the curve (AUC) was calculated. The AUC was $\log _{10}$ transformed to normalize the data, and linear regression was used to assess the treatment effects on residual disease growth (log AUC). Survival time is defined as the length of time between the day of tumor cell injection and the time the mice were found dead or sacrificed due to residual disease burden. Due to the large variability in survival time between groups, linear regression was performed on the $\log _{10}$ of survival time. Analyses were performed using Statistical Package for the Social Sciences version 17.0 (SPSS Inc., Chicago, IL) and Stata 11 (StataCorp LP., College Station, TX). The p-values refer to two-sided tests and a p-value $<0.05$ was considered significant.

\section{RESULTS}

\section{Dinutuximab induces antibody-dependent cell-mediated cytotoxicity when combined with aNK cells in vitro}

GD2 expression levels for all cell lines were assessed by flow cytometry (Supplementary Fig. S1). Each of the three cell lines used for the in vitro assays had greater than $97.9 \%$ of cells with GD2 expression. To assess cytotoxicity in vitro, NB cell lines SH-SY5Y, CHLA-136-Fluc and CHLA-255-Fluc were not treated (control) or treated with dinutuximab alone, aNK cells alone, or the combination of dinutuximab and aNK cells. For all three cell lines, neither dinutuximab nor aNK cells alone at a low effector:target (E:T) cell ratio of 1:2 decreased survival compared to control. The difference between aNK cells combined with dinutuximab and aNK cells alone suggested a trend but did not reach statistical significance. However, the combination of dinutuximab and aNK cells (1:2 E:T ratio) induced a significant decrease in cell viability in all three cell lines compared to control $(\mathrm{p}<0.03$ for each cell line) (Fig. 1). These findings demonstrate cytotoxic effect of aNK cells on NB cells via ADCC when combined with dinutuximab.

\section{Dinutuximab combined with aNK cells inhibits invasion of NB cells in vitro}

An important hallmark of malignancy is the ability to invade the extracellular matrix in order to migrate to distant sites. To test whether aNK cells and/or dinutuximab affect invasiveness in vitro, a Matrigel assay was performed using SH-SY5Y-Fluc, CHLA-136-Fluc and CHLA-255-Fluc NB cells and control medium, dinutuximab alone, aNK cells alone, or the combination of dinutuximab and aNK cells. When NB cells were treated with either aNK cells alone or dinutuximab alone, we observed no significant difference in subsequent cellular migration compared to control untreated cells (Fig. 2A and 2B). There was no significant difference in invasion when dinutuximab was combined with aNK cells compared to aNK cells alone. However, the combination of aNK cells and dinutuximab significantly decreased subsequent NB cell invasion of all three cell lines into Matrigel 
compared to control medium (SH-SY5Y-Fluc, $\mathrm{p}=0.045$; CHLA-136-Fluc, $\mathrm{p}=0.045$;

CHLA-255-Fluc, $\mathrm{p}=0.029)$.

\section{Dinutuximab combined with aNK cells inhibits growth of residual NB following surgical resection of the primary tumor}

For the in vivo studies, we utilized a tumor resection model of neuroblastoma, whereby a xenografted kidney is surgically removed one week following tumor cell injection. Our surgical model demonstrates a significant reduction of NB disease burden when comparing pre- and post-resection bioluminescent imaging (Fig 3A). A 95\% reduction in bioluminescent flux (photons/sec) is observed following primary tumor removal $(\mathrm{p}<0.001)$ (Fig. 3B).

The ability of dinutuximab and adoptively transferred aNK cells to inhibit establishment of detectable disease in liver and bone marrow after surgical resection of the kidney with primary NB was determined using CHLA-136-Fluc, CHLA-255-Fluc, and PDX COG$\mathrm{N}-415 \times$ cells (15). Following resection at day seven, mice were randomized to control, dinutuximab alone, aNK cells alone, and aNK cells plus dinutuximab treatment groups. The presence of NB in liver or bone marrow was analyzed by bioluminescence weekly for CHLA-255-Fluc and CHLA-136-Fluc cell lines and by histologic evaluation at sacrifice for the PDX model.

Bioluminescent imaging of mice injected with CHLA-136-Fluc cells revealed disease in the liver of nearly all but only rarely in the femurs of untreated control mice (Fig. 4A and 4B). Treatment with aNK cells alone or in combination with dinutuximab after resection of CHLA-136-Fluc primary tumor caused a significant decrease in the incidence of bioluminescence within the liver when compared to control mice ( $\mathrm{p}<0.01$ for both) (Fig.

4A). The incidence of bioluminescent signal within liver did not significantly decrease when aNK cells and dinutuximab were compared to aNK cells alone. The infrequency of bioluminescence in femurs precluded identifying an effect of treatment on disease in femurs.

In mice xenografted with CHLA-255-Fluc cells, combination therapy after resection significantly decreased the incidence of bioluminescence in both livers and femurs $(\mathrm{p}<$ 0.05) (Fig. 4A and 4B). In contrast, monotherapy with dinutuximab or aNK cells did not decrease bioluminescence in these sites. A statistical difference in the incidence of bioluminescent signal within liver and femur was not observed between groups treated with aNK cells and dinutuximab versus aNK cells alone.

For mice xenografted with COG-N-415x cells, liver and bone marrow were evaluated by histology for the presence of NB following sacrifice. Similar to findings for CHLA-255Fluc, there was a significant reduction in the incidence of NB present in liver and bone marrow by combination treatment with dinutuximab and aNK cells $(\mathrm{p}<0.05)($ Fig. 4E and $4 F)$. Monotherapy with dinutuximab or aNK cells did not significantly decrease detectable NB in liver or bone marrow.

In addition to evaluating the incidence of distant tumor growth, we also assessed the overall burden or quantity of residual disease in mice xenografted with CHLA-136-Fluc and 
CHLA-255-Fluc cells. We measured the burden of residual disease by measuring total luminescence from each mouse weekly (Fig. 5A). In mice xenografted with CHLA-136-Fluc and CHLA-255-Fluc cells, we observed a significant decrease in residual disease burden after treatment with aNK cells alone or with the combination of aNK cells and dinutuximab as compared to control (Fig. 5B). No significant difference was observed when comparing mice treated with aNK cells alone compared to combination therapy. Together, these data demonstrate the positive effect of aNK cells alone and in combination with dinutuximab in reducing the incidence and burden of distant disease following surgical resection of the primary tumor.

\section{Dinutuximab combined with aNK cells increases survival following surgical resection of the primary tumor}

In the resection model of NB, survival of mice xenografted with CHLA-136-Fluc cells was significantly increased following aNK cells alone with a 16 day increase in mean survival ( $p$ $<0.001)$. The combination of aNK cells and dinutuximab further increased survival compared to both control (mean survival 83.1 versus 51.6 days) and aNK cells alone (mean survival 83.1 versus $71.2 ; \mathrm{p}<0.05$ for both) (Fig. 6). Survival of mice xenografted with CHLA-255-Fluc cells was not increased in the group receiving aNK cells alone compared to control mice but was significantly increased in mice treated with the combination of dinutuximab and aNK cells compared to control (mean survival increased by 13.8 days; $p=$ 0.015). In mice xenografted with COG-N-415x cells, monotherapy with dinutuximab increased mean survival by 4.8 days and aNK cells increased mean survival by 5.3 days as compared to control ( $\mathrm{p}<0.05$ for both) (Fig. 6). Moreover, survival of mice xenografted with COG-N-415 $\times$ cells was greatest in the group receiving the combination of dinutuximab with aNK cells, with a 10.4 day increase in mean survival compared to control ( $\mathrm{p}<0.001)$. Dinutuximab combined with aNK cells significantly increased survival over aNK cells alone following the resection of primary tumors created by both CHLA-136-Fluc and COG$\mathrm{N}-415 \times$ cells. These data demonstrate that following resection of the primary NB, treatment with aNK cells alone increased survival of mice with two of three xenograft models but that the combination dinutuximab and aNK cells was most effective, prolonging survival of mice with all three xenograft models.

\section{DISCUSSION}

We hypothesized that disease control after surgical resection of the primary tumor in patients with high-risk NB can be improved by treatment with adoptively transferred aNK cells and anti-GD2 dinutuximab. To test this hypothesis, we developed a model of high-risk NB using immune deficient NSG mice. Following surgical resection of the primary tumor formed in the kidney by human NB cell lines CHLA-136-Fluc or CHLA-255-Fluc or PDX COG$\mathrm{N}-415 \times$, we show that survival of mice is most effectively increased by adoptive transfer of human aNK cells with dinutuximab. These data support consideration of therapy with adoptively transferred aNK cells and dinutuximab after surgical disease reduction.

Our model generates primary human NBs in the kidney of NSG mice that can be surgically removed, which increases survival (11). Although we show that surgery removes $95 \%$ of 
disease, residual NB cells can be detected consistently with bioluminescent imaging and histopathology in liver and bone marrow, which are sites of disease in patients. We demonstrate that treatment with aNK cells combined with dinutuximab after resection decreases the incidence and quantity of detectable NB within liver and bone marrow and, importantly, increases survival of mice.

Several mechanisms could contribute to the eradication of residual NB cells in primary and distant sites after surgical resection. First, circulating NB cells could be decreased by direct cytotoxicity of aNK cells alone or by ADCC mediated by dinutuximab and aNK cells leaving fewer available to invade into tissues $(24,25)$. In this regard, aNK cells mediate ADCC against NB cells at a relatively low 1:2 E:T cell ratio. Second, NB cells that survive aNK cell-mediated ADCC may have a decreased ability to invade tissues as we have shown that their invasion into Matrigel in vitro is impaired. However, the relative contributions of these and other possible cellular and molecular mechanisms to eradication of residual disease are not currently known.

Although decreased disseminated growth and improved survival could be due to reduction of functionally invasive NB cells, tumor cells may already have become established within the liver and/or bone marrow at the time of surgical resection. If so, immunotherapy would need to be effective against disease in these sites, which requires localization and anti-tumor cell functions of dinutuximab and aNK cells within potentially immune suppressive microenvironments. Indeed, using these same models in NSG mice without resection, we have found that TGF $\beta 1$, which is produced by NB cells, inhibits function of aNK cells together with dinutuximab in the tumor microenvironment (17). Thus, targeting immune suppressive activities with agents such as galunisertib, which inhibits signaling induced by TGF $\beta 1$, could further improve antibody-mediated immunotherapy of NB cells that disseminated before surgical resection.

We demonstrated that dinutuximab combined with adoptively transferred human aNK cells following surgical resection is consistently effective in improving survival of mice using two human NB cell lines and a PDX. Notably, aNK cells alone improved survival for mice with two of the three NB xenografts, suggesting that their interaction with ligands on NB cells can result in cytotoxicity. The NB xenografts used in these experiments are representative of high-risk disease as they were derived from extremely aggressive and drug-resistant metastatic tumors. The CHLA-136 cell line is $M Y C N$-amplified while the CHLA-255 cell line overexpresses c-Myc protein, both of which are indicative of a poor prognosis $(20,26)$. The PDX COG-N-415× cells have $M Y C N$-amplification and an $A L K$ mutation, both of which contribute to aggressive tumor behavior (27). In our experiments, mice were treated with dinutuximab, which is currently a standard of care after myeloablative therapy for patients with high-risk NB. aNK cells are propagated and activated ex vivo similarly to open and upcoming clinical trials (NCT03420963, NCT01853358, NCT03242603, NCT01947322, and NCT02763475). Thus, our model of surgical resection followed by immunotherapy is relevant to the treatment of a spectrum of high-risk NBs in patients.

Although our experiments mimic immunotherapy in patients with high-risk NB, they have possible limitations. First, aNK and NB cells were from unrelated donors and so potentially 
do not provide models for the binding of human leukocyte antigen (HLA) class I molecules by autologous inhibitory and activating killer cell immunoglobulin-like receptors (KIRs) (28). However, aNK cells generated with K562-mbIL21 stimulator cells are not inhibited by autologous KIR-ligand interaction (22). Another possible limitation is absence of effector T cells. However, NB cells express little or no MHC class I molecules to present peptides to effector $\mathrm{T}$ cells and have few gene mutations to generate unique tumor associated antigens/ peptides that could be targets for cytotoxic T cells (29-31). Evidence has not been obtained to date showing an adaptive T cell immune response to human NB (32). These potential limitations could be evaluated in fully immune competent mice with transgenic or transplantable syngeneic NB tumors that express GD2 or other target antigens for antibodies and that have the spectrum of oncogenic abnormalities associated with aggressive tumor behavior in humans, but such models are not currently available.

In summary, we show that dinutuximab combined with aNK cells mediates ADCC against $\mathrm{NB}$ cells and inhibits their invasion in vitro. Importantly, we demonstrate that mouse survival after surgical resection of primary tumors formed by aggressive human NB cells is improved by adoptive transfer of human aNK cells combined with dinutuximab. These data provide support for clinical testing of NK cells that are activated ex vivo or in vivo and combined with dinutuximab for treatment of residual disease after surgical resection of the primary tumor in children with high-risk NB.

\section{Supplementary Material}

Refer to Web version on PubMed Central for supplementary material.

\section{Acknowledgments}

Funding: This research was supported by Children's Hospital Los Angeles Institutional Start-up Funding (ESK) and in part by grant 1 P01 CA217959 from the National Cancer Institute (RCS).

\section{REFERENCES}

1. Handgretinger R, Anderson K, Lang P, Dopfer R, Klingebiel T, Schrappe M, et al. A phase I study of human/mouse chimeric antiganglioside GD2 antibody ch14.18 in patients with neuroblastoma. Eur J Cancer. 1995;31A:261-7. [PubMed: 7718335]

2. Matthay KK, Villablanca JG, Seeger RC, Stram DO, Harris RE, Ramsay NK, et al. Treatment of high-risk neuroblastoma with intensive chemotherapy, radiotherapy, autologous bone marrow transplantation, and 13-cis-retinoic acid. Children's Cancer Group. N Engl J Med. 1999;341:116573. [PubMed: 10519894]

3. Matthay KK, Reynolds CP, Seeger RC, Shimada H, Adkins ES, Haas-Kogan D, et al. Long-term results for children with high-risk neuroblastoma treated on a randomized trial of myeloablative therapy followed by 13-cis-retinoic acid: a children's oncology group study. J Clin Oncol. 2009;27:1007-13. [PubMed: 19171716]

4. Yalcin B, Kremer LC, van Dalen EC. High-dose chemotherapy and autologous haematopoietic stem cell rescue for children with high-risk neuroblastoma. Cochrane Database Syst Rev. 2015:Cd006301. [PubMed: 26436598]

5. Berthold F, Boos J, Burdach S, Erttmann R, Henze G, Hermann J, et al. Myeloablative megatherapy with autologous stem-cell rescue versus oral maintenance chemotherapy as consolidation treatment in patients with high-risk neuroblastoma: a randomised controlled trial. Lancet Oncol. 2005;6:64958. [PubMed: 16129365] 
6. Cheung NK, Kushner BH, Kramer K. Monoclonal antibody-based therapy of neuroblastoma. Hematol Oncol Clin North Am. 2001;15:853-66. [PubMed: 11765377]

7. Franks LM, Bollen A, Seeger RC, Stram DO, Matthay KK. Neuroblastoma in adults and adolescents: an indolent course with poor survival. Cancer. 1997;79:2028-35. [PubMed: 9149032]

8. Kreissman SG, Seeger RC, Matthay KK, London WB, Sposto R, Grupp SA, et al. Purged versus non-purged peripheral blood stem-cell transplantation for high-risk neuroblastoma (COG A3973): a randomised phase 3 trial. Lancet Oncol. 2013;14:999-1008. [PubMed: 23890779]

9. Yu AL, Gilman AL, Ozkaynak MF, London WB, Kreissman SG, Chen HX, et al. Anti-GD2 antibody with GM-CSF, interleukin-2, and isotretinoin for neuroblastoma. N Engl J Med. 2010;363:1324-34. [PubMed: 20879881]

10. Liu Y, Wu HW, Sheard MA, Sposto R, Somanchi SS, Cooper LJ, et al. Growth and activation of natural killer cells ex vivo from children with neuroblastoma for adoptive cell therapy. Clin Cancer Res. 2013;19:2132-43. [PubMed: 23378384]

11. Jackson JR, Kim Y, Seeger RC, Kim ES. A novel minimal residual disease model of neuroblastoma in mice. J Pediatr Surg. 2016;51:991-4. [PubMed: 26995512]

12. Ara T, Nakata R, Sheard MA, Shimada H, Buettner R, Groshen SG, et al. Critical role of STAT3 in IL-6-mediated drug resistance in human neuroblastoma. Cancer Res. 2013;73:3852-64. [PubMed: 23633489]

13. Borriello L, Nakata R, Sheard MA, Fernandez GE, Sposto R, Malvar J, et al. Cancer-Associated Fibroblasts Share Characteristics and Protumorigenic Activity with Mesenchymal Stromal Cells. Cancer Res. 2017;77:5142-57. [PubMed: 28687621]

14. Keshelava N, Zuo JJ, Chen P, Waidyaratne SN, Luna MC, Gomer CJ, et al. Loss of p53 function confers high-level multidrug resistance in neuroblastoma cell lines. Cancer Res. 2001;61:6185-93. [PubMed: 11507071]

15. Keshelava N, Davicioni E, Wan Z, Ji L, Sposto R, Triche TJ, et al. Histone deacetylase 1 gene expression and sensitization of multidrug-resistant neuroblastoma cell lines to cytotoxic agents by depsipeptide. J Natl Cancer Inst. 2007;99:1107-19. [PubMed: 17623797]

16. Lopez-Barcons L, Maurer BJ, Kang MH, Reynolds CP. P450 inhibitor ketoconazole increased the intratumor drug levels and antitumor activity of fenretinide in human neuroblastoma xenograft models. Int J Cancer. 2017;141:405-13. [PubMed: 28340497]

17. Tran HC, Wan Z, Sheard MA, Sun J, Jackson JR, Malvar J, et al. TGFbetaR1 Blockade with Galunisertib (LY2157299) Enhances Anti-Neuroblastoma Activity of the Anti-GD2 Antibody Dinutuximab (ch14.18) with Natural Killer Cells. Clin Cancer Res. 2017;23:804-13. [PubMed: 27756784]

18. Asgharzadeh S, Pique-Regi R, Sposto R, Wang H, Yang Y, Shimada H, et al. Prognostic significance of gene expression profiles of metastatic neuroblastomas lacking MYCN gene amplification. J Natl Cancer Inst. 2006;98:1193-203. [PubMed: 16954472]

19. Asgharzadeh S, Salo JA, Ji L, Oberthuer A, Fischer M, Berthold F, et al. Clinical significance of tumor-associated inflammatory cells in metastatic neuroblastoma. J Clin Oncol. 2012;30:3525-32. [PubMed: 22927533]

20. Wang LL, Teshiba R, Ikegaki N, Tang XX, Naranjo A, London WB, et al. Augmented expression of MYC and/or MYCN protein defines highly aggressive MYC-driven neuroblastoma: a Children's Oncology Group study. Br J Cancer. 2015;113:57-63. [PubMed: 26035700]

21. Eggert A, Grotzer MA, Ikegaki N, Liu XG, Evans AE, Brodeur GM. Expression of the neurotrophin receptor TrkA down-regulates expression and function of angiogenic stimulators in SH-SY5Y neuroblastoma cells. Cancer Res. 2002;62:1802-8. [PubMed: 11912158]

22. Denman CJ, Senyukov VV, Somanchi SS, Phatarpekar PV, Kopp LM, Johnson JL, et al. Membrane-Bound IL-21 Promotes Sustained Ex Vivo Proliferation of Human Natural Killer Cells. PloS one. 2012;7:e30264. [PubMed: 22279576]

23. Keshelava N, Frgala T, Krejsa J, Kalous O, Reynolds CP. DIMSCAN: a microcomputer fluorescence-based cytotoxicity assay for preclinical testing of combination chemotherapy. Methods Mol Med. 2005;110:139-53. [PubMed: 15901933]

24. Hanna N, Fidler IJ. Role of natural killer cells in the destruction of circulating tumor emboli. J Natl Cancer Inst. 1980;65:801-9. [PubMed: 6932529] 
25. Langers I, Renoux VM, Thiry M, Delvenne P, Jacobs N. Natural killer cells: role in local tumor growth and metastasis. Biologics. 2012;6:73-82. [PubMed: 22532775]

26. Seeger RC, Brodeur GM, Sather H, Dalton A, Siegel SE, Wong KY, et al. Association of multiple copies of the N-myc oncogene with rapid progression of neuroblastomas. N Engl J Med. 1985;313:1111-6. [PubMed: 4047115]

27. De Brouwer S, De Preter K, Kumps C, Zabrocki P, Porcu M, Westerhout EM, et al. Meta-analysis of neuroblastomas reveals a skewed ALK mutation spectrum in tumors with MYCN amplification. Clin Cancer Res. 2010;16:4353-62. [PubMed: 20719933]

28. Erbe AK, Wang W, Carmichael L, Kim K, Mendonca EA, Song Y, et al. Neuroblastoma Patients' KIR and KIR-Ligand Genotypes Influence Clinical Outcome for Dinutuximab-based Immunotherapy: A Report from the Children's Oncology Group. Clin Cancer Res. 2018;24:189_ 96. [PubMed: 28972044]

29. Pugh TJ, Morozova O, Attiyeh EF, Asgharzadeh S, Wei JS, Auclair D, et al. The genetic landscape of high-risk neuroblastoma. Nat Genet. 2013;45:279-84. [PubMed: 23334666]

30. Main EK, Lampson LA, Hart MK, Kornbluth J, Wilson DB. Human neuroblastoma cell lines are susceptible to lysis by natural killer cells but not by cytotoxic T lymphocytes. J Immunol. 1985;135:242-6. [PubMed: 3158702]

31. Prigione I, Corrias MV, Airoldi I, Raffaghello L, Morandi F, Bocca P, et al. Immunogenicity of human neuroblastoma. Ann N Y Acad Sci. 2004;1028:69-80. [PubMed: 15650233]

32. Majzner RG, Heitzeneder S, Mackall CL. Harnessing the Immunotherapy Revolution for the Treatment of Childhood Cancers. Cancer Cell. 2017;31:476-85. [PubMed: 28366678] 


\section{TRANSLATIONAL RELEVANCE}

Children with high-risk neuroblastoma often achieve clinically defined remission during or following induction phase chemotherapy and surgery, consolidation phase myeloablative chemotherapy, and post-consolidation phase antibody immunotherapy. Overall, $45 \%$ become long-term survivors, but the remainder develop refractory or recurrent disease, and nearly all subsequently succumb. Immunotherapy with the antiGD2 antibody dinutuximab following myeloablative chemotherapy improves 2-year event-free and overall survival. Dinutuximab, when combined with chemotherapy, also can be effective against persistent or recurrent disease. Experimentally, dinutuximab with ex vivo activated natural killer (aNK) cells causes antibody-dependent cellular cytotoxicity in vitro and tumor responses in mice. This combination has not been examined experimentally or clinically for efficacy after surgical removal of the primary tumor. Our pre-clinical study demonstrates that treatment with the combination of dinutuximab and aNK cells after surgical resection of bulk disease significantly inhibits residual tumor growth and improves survival. This strategy could further improve survival of children with high-risk neuroblastoma. 


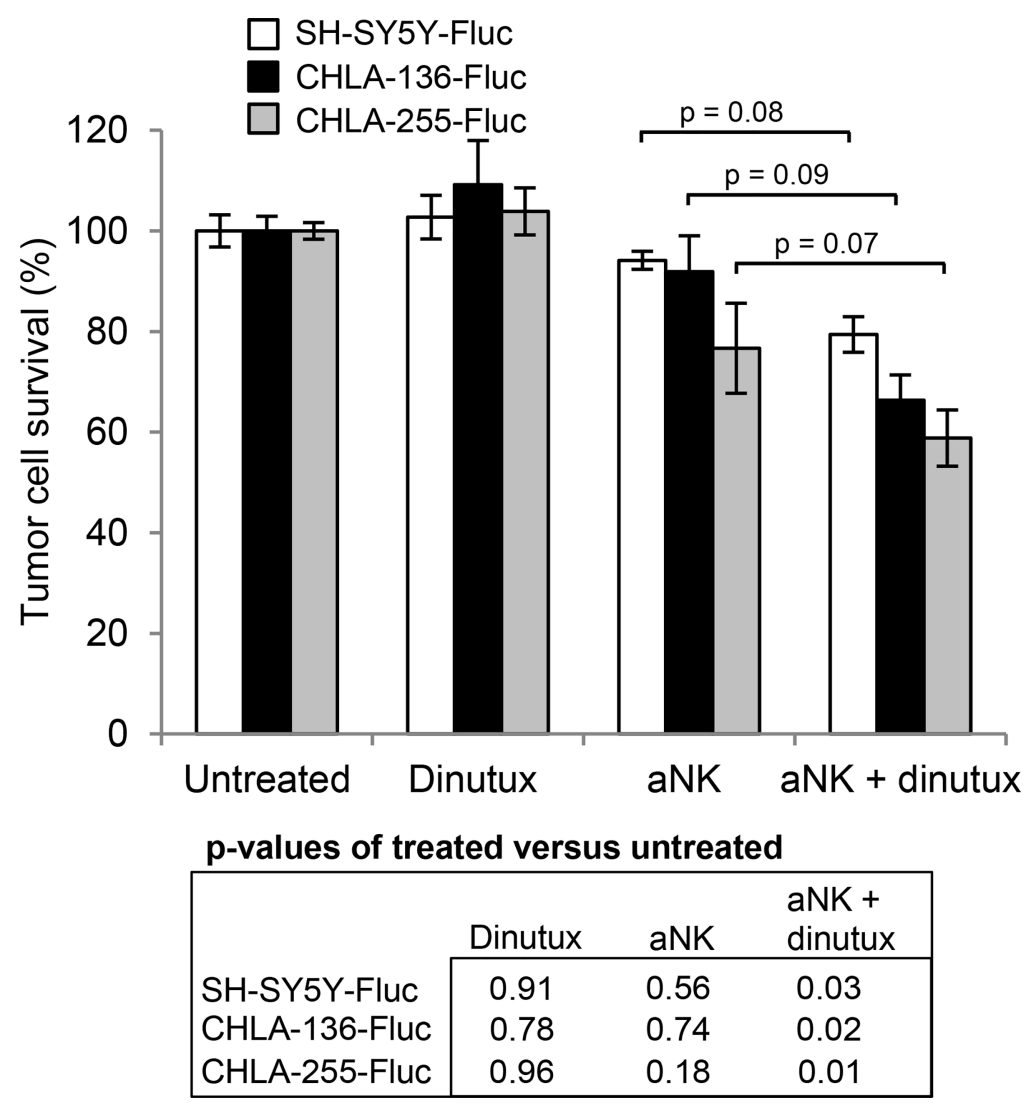

Figure 1.

Effect of anti-GD2 antibody dinutuximab (dinutux) and aNK cells on neuroblastoma cells in vitro. Calcein AM-based digital imaging microscopy assay showing the viability of human neuroblastoma cell lines SH-SY5Y-Fluc, CHLA-255-Fluc and CHLA-136-Fluc following treatment with dinutuximab alone $(100 \mathrm{ng} / \mathrm{ml})$, aNK cells alone, or their combination for six hours. For each cell line, $3 \times 10^{4} \mathrm{NB}$ cells were seeded per well of a 96-well plate, along with $1.5 \times 10^{4}$ aNK cells as described in Materials \& Methods (E:T ratio 1:2). Each condition contained six replicates and each experiment was performed a minimum of two times. Comparisons were made between groups using ANOVA and post-hoc Tukey's HSD tests. 

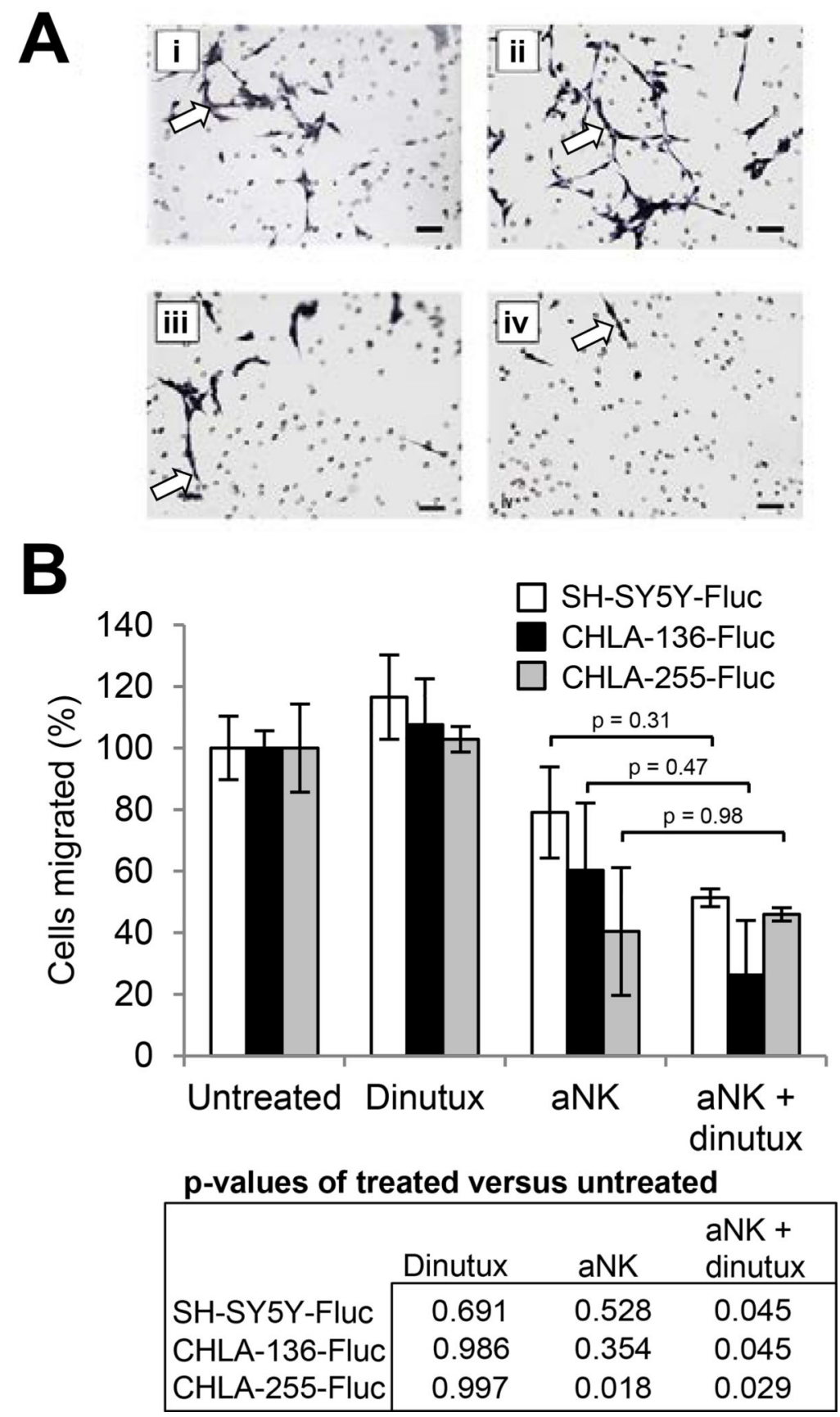

Figure 2.

Effect of activated NK cells and dinutuximab on neuroblastoma cell invasion in vitro. Neuroblastoma cell lines SH-SY5Y-Fluc, CHLA-255-Fluc and CHLA-136-Fluc were treated with vehicle control, dinutuximab alone $(100 \mathrm{ng} / \mathrm{ml})$, aNK cells alone (E:T ratio 1:1 maintained with $100 \mathrm{U} / \mathrm{ml}$ of IL-2), or dinutuximab and aNK in combination. $1 \times 10^{5} \mathrm{NB}$ cells from each treatment group were seeded into the upper chamber of a Biocoat 24-well Matrigel Invasion Chamber (Corning), incubated for 72 hours, and then cells that migrated to the lower chamber were quantified. (A) Photomicrographs of SH-SY5Y-Fluc cells which traversed the Matrigel interface, from the following treatment groups: (i) untreated control, 
(ii) dinutuximab, (iii) aNK cells and (iv) aNK cells + dinutuximab. NB cells appear dark and elongated (arrows) while the small round circles are the microscopic pores in the Matrigel. Scale bar, $100 \mu \mathrm{m}$. (B) Percent of migrated NB cells following treatment with dinutuximab alone, aNK cells alone, or combination treatment. Comparisons were made between groups using ANOVA and post-hoc Tukey's HSD tests. 

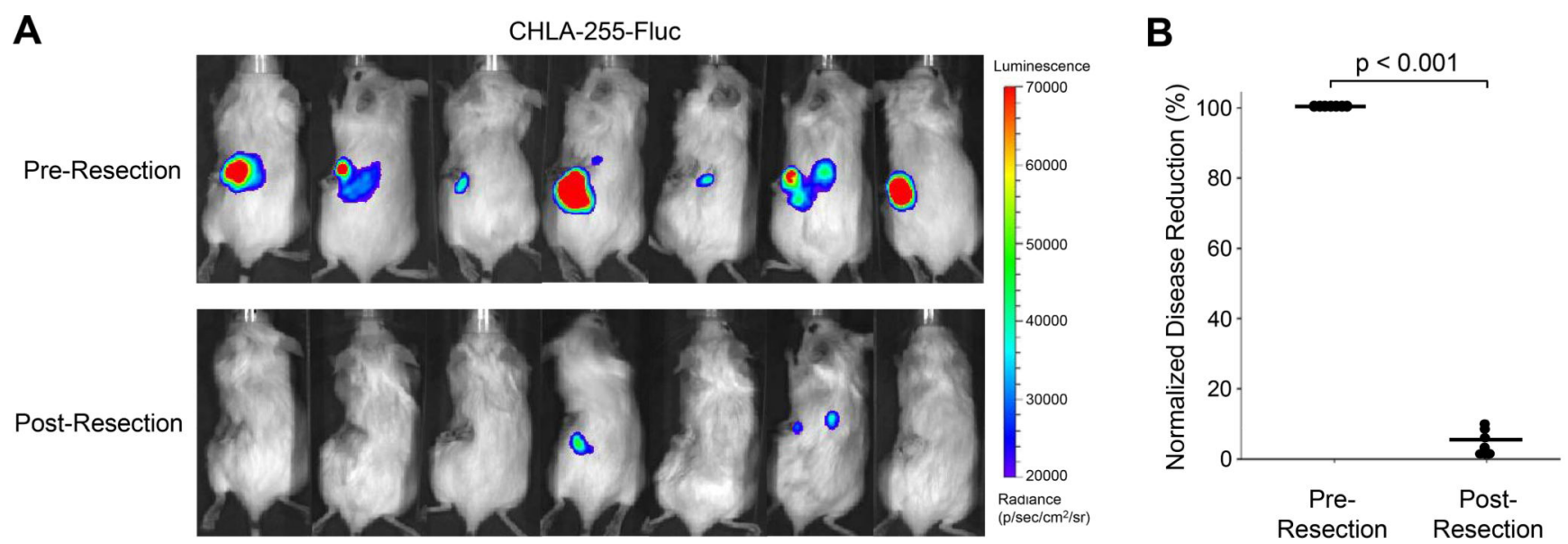

Figure 3.

Disease reduction following surgical resection of primary NB. Mice underwent implantation of $1 \times 10^{6}$ CHLA-255-Fluc NB cells followed by surgical removal of the primary tumor one week later. (A) Bioluminescent images taken 7 days post NB cell injection and just prior to tumor removal. Post-resection images of mice were taken one day after resection and compared to pre-resection baseline. (B) Surgical resection of the primary tumor resulted in a $95 \%$ reduction in disease burden. 


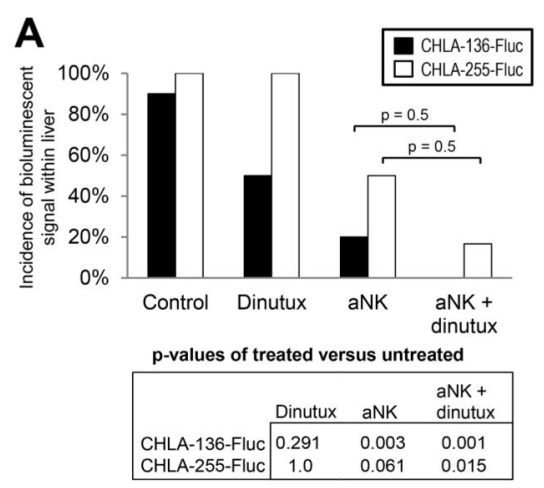

B

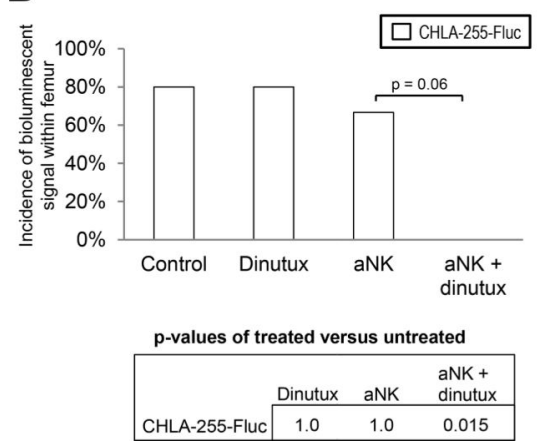

C

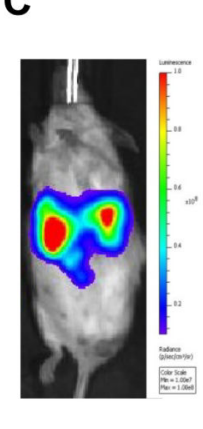

D

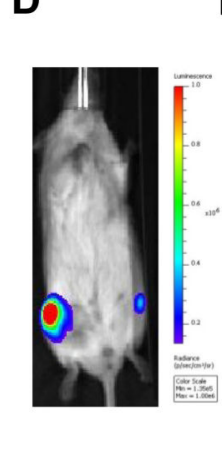

E

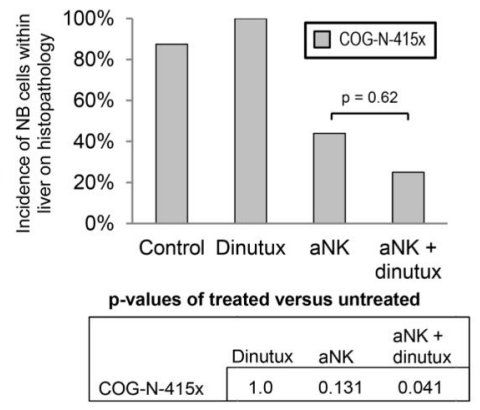

$\mathbf{F}$

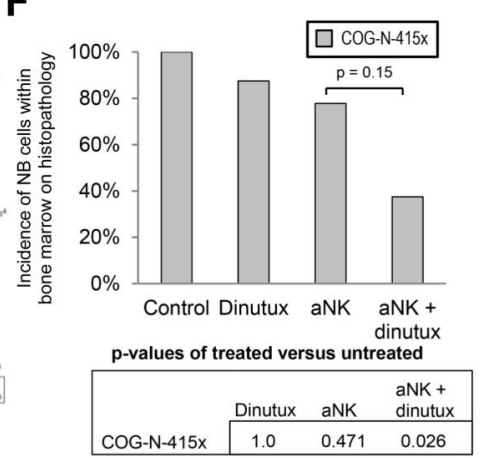

G

COG-N-415x

Liver

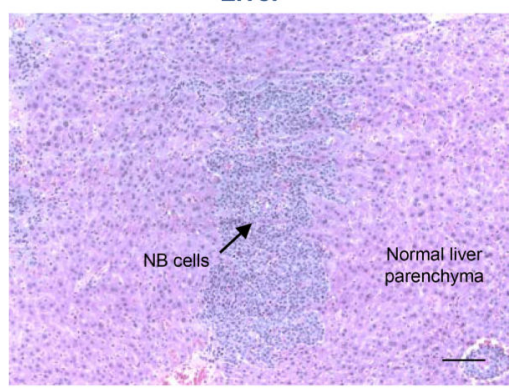

$\mathbf{H}$

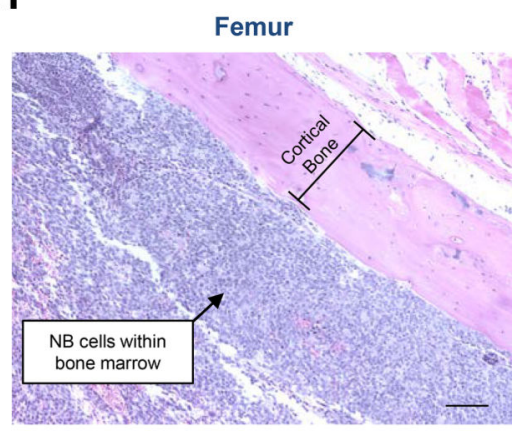

Figure 4.

Effect of activated NK cells combined with dinutuximab on residual NB in vivo. A surgical resection model of NB was utilized, in which mice were injected with $1 \times 10^{6} \mathrm{NB}$ cells (CHLA-136-Fluc, $n=40$; CHLA-255-Fluc, $n=22$; COG-N-415 $\times, n=33$ ) into the left kidney, followed by complete resection of the xenografted kidney one week later. Mice were then randomized into 4 treatment groups: (i) control, (ii) dinutuximab (15 $\mu \mathrm{g} / \mathrm{mouse}$ ), (iii) $1 \times 10^{7}$ aNK cells or (iv) $1 \times 10^{7}$ aNK cells and dinutuximab. Mice receiving aNK cells were given IL-2 ( $2 \mu \mathrm{g} / \mathrm{mouse})$ and IL-15 (5 $\mu \mathrm{g} / \mathrm{mouse})$ via intraperitoneal injection. All treatments were administered twice a week for a total of 4 weeks. (A,B) Incidence of residual NB tumor growth following resection of CHLA-136-Fluc and CHLA-255-Fluc xenografts as determined by the presence of bioluminescent signals in the liver or femurs. CHLA-135Fluc cells were rarely found within the bone marrow and therefore not shown. (C) Bioluminescent imaging of residual disease within the liver of an untreated mouse following resection of CHLA-136-Fluc xenograft. (D) Signal from the femurs of a mouse treated with dinutuximab alone following resection of CHLA-255-Fluc xenograft. (E,F) Incidence of residual tumor growth following resection of COG-N-415x xenograft as determined by histopathology at the time of necropsy. (G,H) H\&E staining, demonstrating NB cells (arrow) within the liver and bone marrow following resection of COG-N-415x xenograft. Scale bar, $200 \mu \mathrm{m}$. 
CHLA-136-Fluc

A

A

Pre-Treatment

Post-Treatment

CHLA-255-Fluc

Untreated

[inimini:

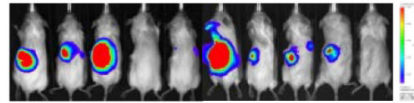

Pre-Treatment

Post-Treatment

Dinutux
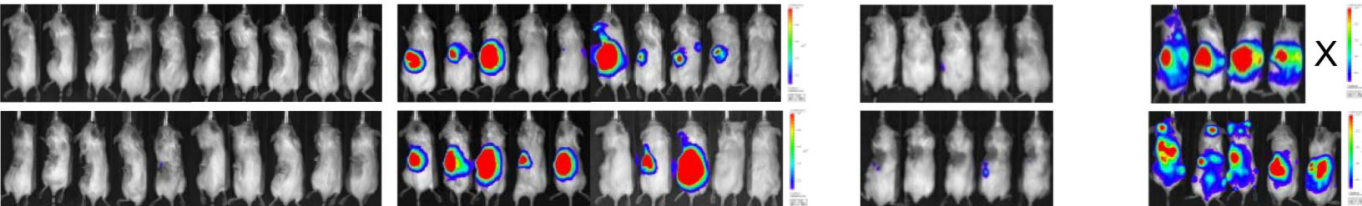

aNK
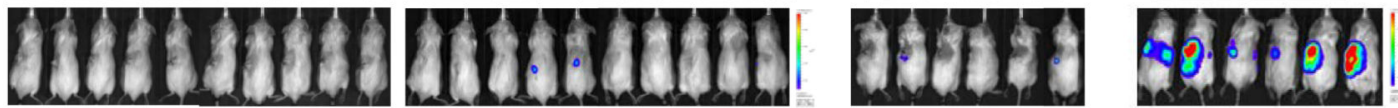

aNK + dinutux
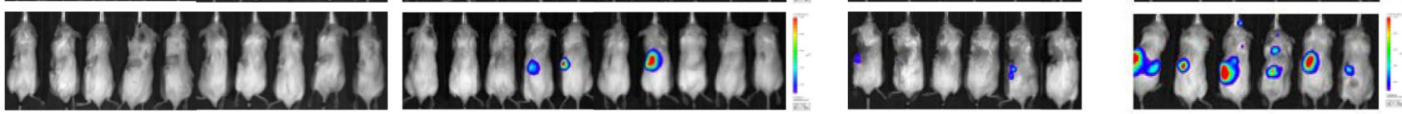

B
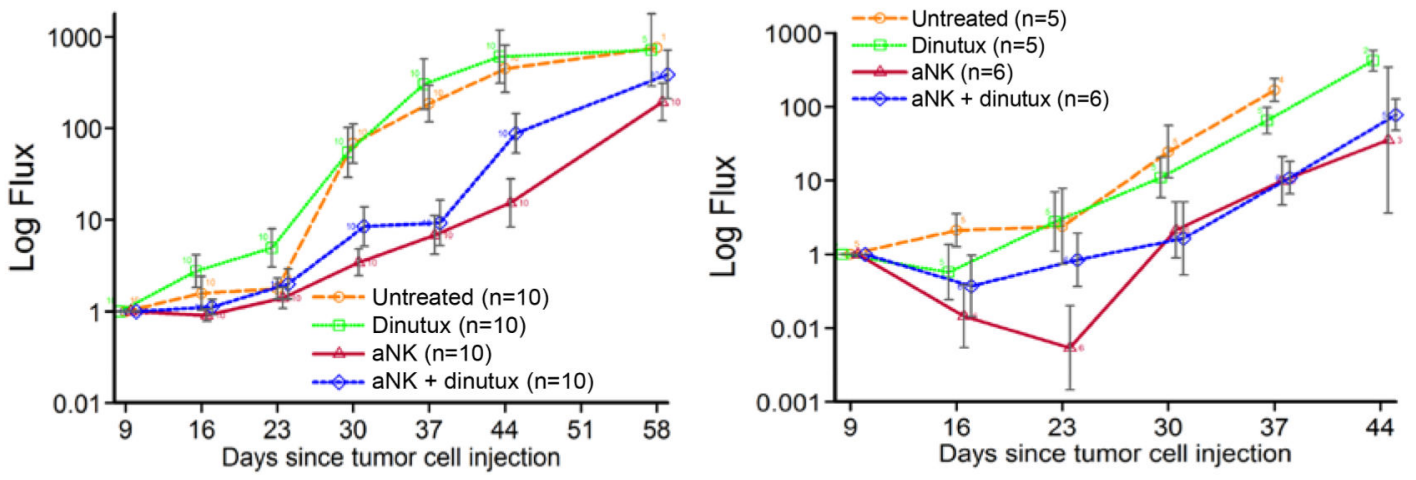

CHLA-136-Fluc p-values

\begin{tabular}{|lccc|}
\hline \multirow{4}{*}{} & Dinutux & aNK & $\begin{array}{l}\text { aNK }+ \\
\text { dinutux }\end{array}$ \\
\cline { 2 - 4 } Untreated & 0.331 & 0.001 & 0.009 \\
Dinutux & $\mathrm{x}$ & 0.001 & 0.001 \\
aNK & $\mathrm{x}$ & $\mathrm{x}$ & 0.180 \\
\hline
\end{tabular}

\begin{tabular}{lccc|}
\multicolumn{4}{l}{ CHLA-255-Fluc $\mathrm{p}$-values } \\
\cline { 2 - 4 } & Dinutux & aNK & $\begin{array}{l}\text { aNK + } \\
\text { dinutux }\end{array}$ \\
\cline { 2 - 4 } Untreated & 0.318 & 0.007 & 0.012 \\
Dinutux & $\mathrm{x}$ & 0.055 & 0.091 \\
aNK & $\mathrm{x}$ & $\mathrm{x}$ & 0.792 \\
\hline
\end{tabular}

Figure 5.

aNK cells alone and in combination with dinutuximab decrease overall disease burden following primary tumor resection. (A) Images of disease burden prior to treatment and 2 days after the completion of treatment are shown from mice following resection of primary tumors created by CHLA-136-Fluc and CHLA-255-Fluc cells. (B) Bioluminescent imaging was performed weekly and tumor flux (photons/sec) measurements were adjusted to baseline observations. The area under the curve (AUC) was calculated and linear regression was used to assess treatment effects. 

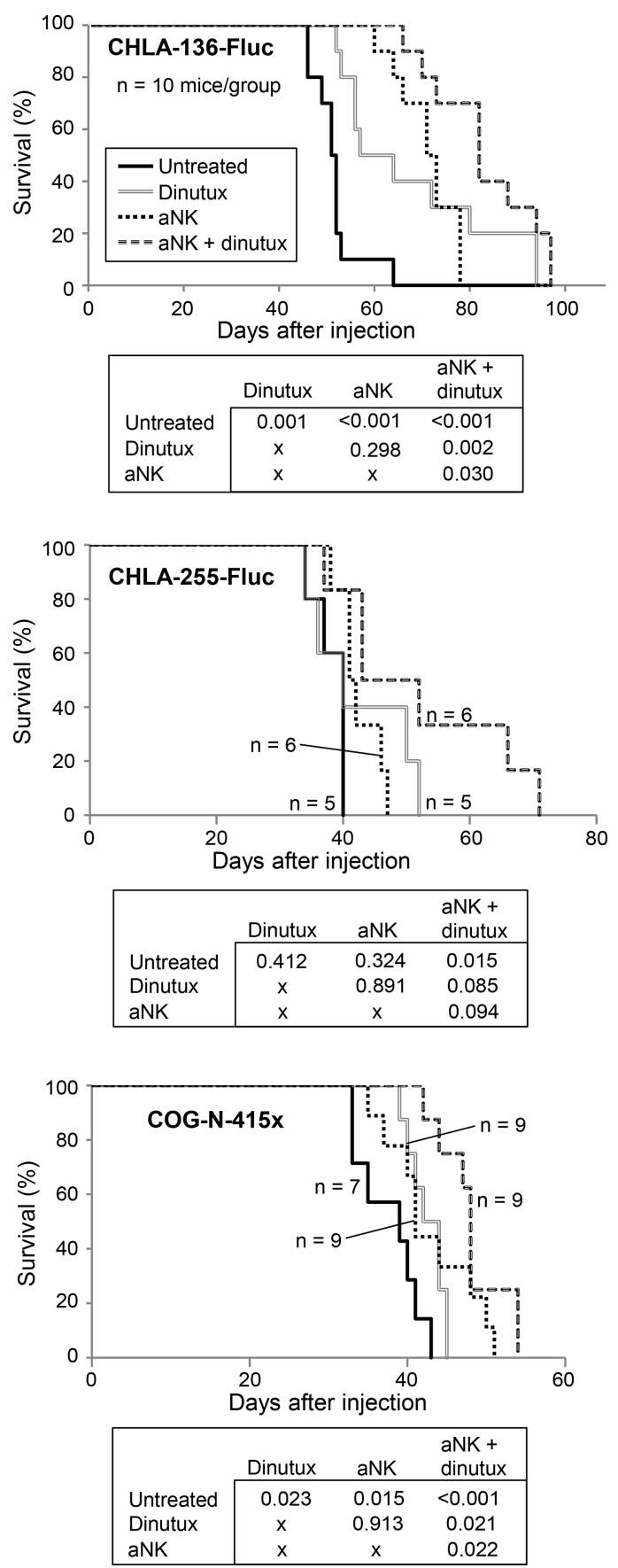

Figure 6.

aNK cells and dinutuximab improve survival following surgical resection of primary tumors created from CHLA-136-Fluc, CHLA-255-Fluc and COG-N-415×. Survival plots shown here and the differences in survival between groups was analyzed with linear regression. 